\title{
Deep inelastic sum rules at the boundaries between perturbative and non-perturbative QCD
}

\author{
A.L. Kataev ${ }^{1}$ \\ Institute for Nuclear Research of the Russian Academy of Sciences , \\ 117312 Moscow, Russsia
}

\begin{abstract}
The basis of renormalon calculus is briefly discussed. This method is applied to study the QCD predictions for three different sum rules of deep-inelastic scattering, namely for the Gross-Llewellyn Smith, Bjorken polarized and unpolarized sum rules. It is shown that the renormalon structures of these a posteriori different physical quantities are closely related. These properties are giving us the hint that theoretical expressions of these three sum rules are similar both in the perturbative and non-perturbative sectors. Some phenomenological consequences of the new relations are discussed.
\end{abstract}

PACS: 12.38.Bx;12.38.Cy; 13.85.Hd

Keywords: Perturbation theory; Renormalons; Deep-inelastic scattering sum rules

\footnotetext{
${ }^{1}$ E-mail:kataev@ms2.inr.ac.ru
} 


\section{Introduction}

The formulation of the quantitative method of renormalon calculus on the higher level of understanding takes its start from the important works of Ref. 1, 2], devoted to the consideration of $e^{+} e^{-} \rightarrow$ hadrons process, and from the interesting work of Ref. [3], devoted to the consideration of deep-inelastic scattering processes.

After these studies the number of theoretical and practical developments appeared in the literature (see reviews of Refs. [4]-6] ).

In what is discussed below, we will consider aspects of renormalon calculus, related to deep-inelastic scattering (DIS) sum rules.

It is commonly expected that in the canonical renormalization schemes, say the $\overline{\mathrm{MS}}$ scheme, perturbative expansions in small QCD coupling constant $a_{s}=\alpha_{s} /(4 \pi)$ of theoretical expressions for physical quantities, defined in the Euclidean region, are asymptotic ones. This means that the difference of the total sums

$$
D(a)=1+\sum_{n \geq 1} d_{n} a^{n}
$$

and their finite sums

$$
D_{\mathrm{k}}(a)=1+\sum_{n=1}^{\mathrm{k}} d_{n} a^{\mathrm{k}}
$$

satisfy the following property

$$
\lim _{a \rightarrow 0}\left|\frac{D(a)-D_{\mathrm{k}}(a)}{a^{\mathrm{k}}}\right| \rightarrow 0
$$

In other words the difference between the total series and their finite sum are expressed as

$$
D(a)-D_{\mathrm{k}}(a)=O\left(a^{\mathrm{k}+1}\right) .
$$

In this case the error of the truncation of the asymptotic series can be estimated by the last term of $D_{\mathrm{k}}(a)$, namely $d_{\mathrm{k}} a^{\mathrm{k}}[7]$.

In QCD one expects that in the $\overline{\mathrm{MS}}$ scheme the coefficient function for DIS sum rules, normalized to unity can be approximated by the following asymptotic series [8]:

$$
D_{\mathrm{k}}(a)=1+\sum_{\mathrm{k} \geq 1}\left(\beta_{0}\right)^{\mathrm{k}} \mathrm{k} !\left(K_{D}^{\mathrm{UV}}(-1)^{\mathrm{k}} \mathrm{k}^{a}+K_{D}^{\mathrm{IR}} \mathrm{k}^{b}\right) a_{s}^{\mathrm{k}+1}
$$

where sign-alternating series with the coefficient $K^{\mathrm{UV}}$ is generated by the ultraviolet renormalons (UVR), sign-constant asymptotic series with coefficient $K^{\mathrm{IR}}$ result from the consideration of infrared renormalons (IRR), and $a$ and $b$ are the known numbers, that depend from the ratio of the first two coefficients of the QCD $\beta$-function.

Working within renormalon calculus we will demonstrate that the perturbative and non-perturbative contributions to definite DIS sum rules are related. In other words we will show that the renormalon approach is working at the boundaries between these two regimes in QCD.

The aim of this article is three fold: 
- to explain the basic stages of renormalon calculus in QCD, using the simple language;

- to show that in the asymptotic perturbative expansion of three DIS sum rules, namely of the Gross-Llewellyn Smith (GLS), Bjorken- polarized (Bjp) and Bjorkenunpolarized (Bjunp) sum rules, may be universal. We will present arguments, based on the consideration of the results given in Refs. [9, [10, that these expansions are defined by the poles in the closely related Borel images of all three sum rules.

- We will explain the features, which follow from the consideration of the IRR poles in the Borel images of the three DIS sum rules. Moreover our aim is to outline new consequences of the IRR calculus. They indicate the existence of relations between twist-4 $1 / Q^{2}$ non-perturbative contributions to the sum rules we are interested in [11]. These results form the basis of the new QCD relations between theoretical expressions for these three sum rules [11, which seem to be supported by the experimental data within existing error bars. More critical tests of these relations are proposed.

\section{Renormalon calculus and DIS sum rules}

Let us first express a perturbative QCD series in terms of a Borel integral as

$$
\begin{aligned}
D\left(a_{s}\right) & =\sum_{n=0}^{\infty} d_{n} a_{s}^{n} \\
& =\sum_{n=0}^{\infty} d_{n} \frac{n !}{\Gamma(n+1)} \delta^{n} \\
& =\int_{0}^{\infty} \exp \left(-\delta / \beta_{0} a_{s}\right) \sum_{n=0}^{\infty} d_{n} \frac{\delta^{n}}{n !} d \delta \\
& =\int_{0}^{\infty} \exp \left(-\delta / \beta_{0} a_{s}\right) B[D](\delta) d \delta,
\end{aligned}
$$

where $\beta_{0}=(11 / 3) C_{A}-(4 / 3) T_{f} N_{f}$ is the first coefficient of the QCD $\beta$-function, with $C_{A}=3, T_{f}=1 / 2$, and $B[D](\delta)$ is the image of the Borel integral.

At this stage we define the DIS sum rules we will be interested in. The GLS sum rule of the $\nu N$ DIS[12] has the following form

$$
\begin{aligned}
\operatorname{GLS}\left(Q^{2}\right) & =\frac{1}{2} \int_{0}^{1} d x\left[F_{3}^{\nu n}\left(x, Q^{2}\right)+F_{3}^{\nu p}\left(x, Q^{2}\right)\right] \\
& =3 C_{\mathrm{GLS}}\left(Q^{2}\right)-\frac{\left\langle\left\langle O_{1}\right\rangle\right\rangle}{Q^{2}}-O\left(\frac{1}{Q^{4}}\right) .
\end{aligned}
$$

In the Born approximation, this "measures" the number of valence quarks, that are contained in the nucleon and can thus be considered as the baryon sum rule. In the $\overline{\mathrm{MS}}$ scheme, the twist-2 perturbative coefficient function $C_{G L S}\left(Q^{2}\right)$ is calculated explicitly, including $a_{s}^{2}$ and $a_{s}^{3}$ terms [13], 14]. The twist-4 matrix element of the $O\left(1 / Q^{2}\right)$ nonperturbative contribution to the GLS sum rule is related to the matrix element calculated 
in Ref. 15] to be

$$
\left\langle\left\langle O_{1}\right\rangle\right\rangle=\frac{8}{27}\left\langle\left\langle O^{\mathrm{S}}\right\rangle\right\rangle
$$

where $\left\langle\left\langle O^{\mathrm{S}}\right\rangle\right\rangle$ is defined by the following operator

$$
O_{\mu}=\bar{u} \tilde{G}_{\mu \nu} \gamma_{\nu} \gamma_{5} u+(u \rightarrow d)
$$

where

$$
\tilde{G}_{\mu \nu}=\frac{1}{2} \epsilon_{\mu \nu \alpha \beta} G_{\alpha \beta}^{a} \frac{\lambda^{a}}{2}
$$

and

$$
\left\langle P\left|O_{\mu}^{\mathrm{S}}\right| P\right\rangle=2 p_{\mu}\left\langle\left\langle O^{\mathrm{S}}\right\rangle\right\rangle
$$

The second sum rules, actively studied both in theory and experiment, is the Bjp sum rule [16], having the physical meaning of polarized isospin sum rule. Its theoretical expression can be defined as

$$
\begin{aligned}
\operatorname{Bjp}\left(Q^{2}\right) & =\int_{0}^{1} d x\left[g_{1}^{l p}\left(x, Q^{2}\right)-g_{1}^{l n}\left(x, Q^{2}\right)\right] \\
& =\frac{g_{A}}{6} C_{\mathrm{Bjp}}\left(Q^{2}\right)-\frac{\left\langle\left\langle O_{2}\right\rangle\right\rangle}{Q^{2}}-O\left(\frac{1}{Q^{4}}\right) .
\end{aligned}
$$

Here $g_{A}=1.26$ is the known $\beta$-decay constant. At the $a_{s}^{3}$ level its perurbative part differs from the one of the GLS sum rule by the absence of small "light-by-light"-type terms, proportional to the colour structure $d^{a b c} d^{a b c}$ [14]. The structure of the power corrections to the matrix element of the leading $O\left(1 / Q^{2}\right)$ power correction was analytically calculated in Ref. 17, with the useful correction input from the considerations of Ref. 18. The final expressions are presented in a simple-form in the review of Ref.[19], from which we can get:

$$
\left\langle\left\langle O_{2}\right\rangle\right\rangle=\frac{1}{6} \frac{8}{9}\left[\left\langle\left\langle U^{\mathrm{NS}}\right\rangle\right\rangle-\frac{M_{N}^{2}}{4}\left\langle\left\langle V^{\mathrm{NS}}\right\rangle\right\rangle\right]
$$

where

$$
\begin{aligned}
\left\langle P, S\left|U_{\mu}^{\mathrm{NS}}\right| P, S\right\rangle & =2 M_{N} S_{\mu}\left\langle\left\langle U^{\mathrm{NS}}\right\rangle\right\rangle \\
\left\langle P, S\left|V_{\mu \nu, \sigma}^{\mathrm{NS}}\right| P, S\right\rangle & \left.=2 M_{N}\left\langle\left\langle V^{\mathrm{NS}}\right\rangle\right\rangle\left\{\left(S_{\mu} P_{\nu}-S_{\nu} P_{\mu}\right) P_{\delta}\right)\right\}_{S\{\nu, \sigma\}}
\end{aligned}
$$

and $\left\langle\left\langle U^{\mathrm{NS}}\right\rangle\right\rangle$ and $\left\langle\left\langle V^{\mathrm{NS}}\right\rangle\right\rangle$ are the reduced matrix elements of the local operators from Ref.[17], namely

$$
\begin{aligned}
U_{\mu}^{\mathrm{NS}} & =g_{s}\left[\bar{u} \tilde{G}_{\mu, \nu} \gamma^{\nu} u-(u \rightarrow d)\right] \\
V_{\mu \nu, \sigma}^{\mathrm{NS}} & =g_{s}\left\{\bar{u} \tilde{G}_{\mu \nu} \gamma_{\delta} u-(u \rightarrow d)\right\}_{S\{\nu, \delta\}},
\end{aligned}
$$

where $S\{\nu, \sigma\}$ stand for symmetrization over the given subscripts and $\tilde{G}_{\mu, \nu}$ is defined in Eq. (11). In Ref. [20] the definition of Eq. (14) was used for the estimates of $O\left(1 / Q^{2}\right)$ corrections to Bjp sum rule, using the three-point function QCD sum rules technique. These calculations were then re-analyzed with the same method in Ref. 21]. The numerical 
results of these calculations will be discussed later. In the work of Ref.[22] a similar analysis was done with the help of the same method for the first term in the r.h.s. of Eq. (14), while the term, proportional to $\left(M_{N}^{2} / 4\right)\langle\langle V\rangle\rangle$ was included into an $O\left(M_{N}^{2} / Q^{2}\right)$ kinematic $\mathrm{Al}$ power correction to the Bjp sum rule, which involves the second $x^{2}$ moments of the leading-twist contribution to $g_{1}^{p-n}=g_{1}^{p}-g_{1}^{n}$ and the twist-3 matrix element, defined through the combination of $x^{2}$-weighted moments of the difference of structure functions $g_{1}^{p-n}$ and of $g_{2}^{p-n}=g_{2}^{p}\left(x, Q^{2}\right)-g_{2}^{n}\left(x, Q^{2}\right)$ as

$$
d_{2}^{p-n}=\int_{0}^{1} d x x^{2}\left(2 g_{1}^{p-n}\left(x, Q^{2}\right)+3 g_{2}^{p-n}\left(x, Q^{2}\right)\right),
$$

Taking into account this decomposition, it is possible to rewrite a theoretical expression for the numerator of the $1 / Q^{2}$ contribution, in the way it was done say, in the most recent experimental work of Ref. 23 ]

$$
\mu_{4}^{p-n}=\frac{M_{N}^{2}}{9}\left(a_{2}^{p-n}+4 d_{2}^{p-n}+4 f_{2}^{p-n}\right)
$$

where

$$
a_{2}=\int_{0}^{1} d x x^{2}\left[g_{1}^{p}\left(x, Q^{2}\right)-g_{1}^{n}\left(x, Q^{2}\right)\right]
$$

is the target mass correction and

$$
2 m_{N}^{2} f_{2}^{p-n} S_{\mu}=-4 M_{N} S_{\mu}\left\langle\left\langle U^{\mathrm{NS}}\right\rangle\right\rangle
$$

is the twist-4 contribution, which is related to the definition used by us as

$$
\left\langle\left\langle O_{2}\right\rangle\right\rangle=\frac{1}{6} \frac{8}{9}\left\langle\left\langle U^{\mathrm{NS}}\right\rangle\right\rangle=-\frac{1}{6} \frac{4}{9} M_{N}^{2} f_{2}^{p-n} .
$$

In other words we have the following relation

$$
M_{N}^{2} f_{2}^{p-n}=-2\left\langle\left\langle U^{\mathrm{NS}}\right\rangle\right\rangle
$$

It should be stressed that in the region where the perturbative theory is working well enough and the application of the operator-product expansion method is valid (say at $Q^{2} \geq 2 \mathrm{GeV}^{2}$ ), both target mass corrections and twist-3 terms are small and we will neglect them in our further considerations ${ }^{2}$. These features were revealed in the process of the analysis of Ref. [20].

The third sum rule, which was originally derived for purely theoretical purposes, is the Bjorken unpolarized sum rule[24]. It can be written down as:

$$
\begin{aligned}
\operatorname{Bjunp}\left(Q^{2}\right) & =\int_{0}^{1} d x\left[F_{1}^{\nu p}\left(x, Q^{2}\right)-F_{1}^{\nu n}\left(x, Q^{2}\right)\right] \\
& =C_{\text {Bjunp }}\left(Q^{2}\right)-\frac{\left\langle\left\langle O_{3}\right\rangle\right\rangle}{Q^{2}}-O\left(\frac{1}{Q^{4}}\right) .
\end{aligned}
$$

\footnotetext{
${ }^{2}$ For completeness we note that there is a minor difference between the the $O\left(M_{N}^{2} / Q^{2}\right)$ coefficients of the $\int_{0}^{1} d x x^{2} g_{1}^{p-n}$ terms in Ref. [20] and Ref. [23]. In the former and latter cases they are equal to (10/9) and 1 respectively.
} 
It may be also studied in future as the valuable test of QCD both in perturbative and non-perturbative sectors.

As in the previous two cases, the coefficient function $C_{\text {Bjunp }}\left(Q^{2}\right)$ is calculated up to next-to-next-to-leading order $a_{s}^{3}$-corrections [25, 26]. The twist-4 matrix element to this sum rule was evaluated in Ref.[15]; with the following result:

$$
\left\langle\left\langle O_{3}\right\rangle\right\rangle=\frac{8}{9}\left\langle\left\langle O^{\mathrm{NS}}\right\rangle\right\rangle
$$

where the matrix element $\left\langle\left\langle O^{\mathrm{NS}}\right\rangle\right\rangle$ is related to the dimension-5 operator

$$
O_{\mu}^{\mathrm{NS}}=\bar{u} \tilde{G}_{\mu \nu} \gamma_{\nu} \gamma_{5} u-\bar{d} \tilde{G}_{\mu \nu} \gamma_{\nu} \gamma_{5} d
$$

its matching over nucleon states

$$
\left\langle P\left|O_{\mu}^{\mathrm{NS}}\right| P\right\rangle=2 p_{\mu}\left\langle\left\langle O^{\mathrm{NS}}\right\rangle\right\rangle
$$

and application of Eq. (24).

Let us now return to the renormalon calculus. The basic theoretical problem is how to define the Borel image $B[D](\delta)$ (or the Borel sum) of the integral in Eq. (17) for the quantities we are interested in. In QCD this problem is usually solved using perturbative methods and calculating the corresponding multiloop Feynman diagrams with a one-gluon line, dressed by the chains of fermion bubbles (so called renormalon chain insertion). These chains are generating sign-alternating asymptotic perturbative series, typical of the quantities under consideration, in powers of the expansion parameter $N_{f} a_{s}$ (where $N_{f}$ is the number of quarks flavours). The contributions of these chains are gauge-invariant, but they do not reflect the whole picture of renormalon effects in QCD. The latter begin to manifest themselves after application of the naive non-abelianization (NNA) ansatz [27] only, namely after the replacement $N_{f} \rightarrow-(3 / 2) \beta_{0}=N_{f}-(33 / 2)$ in the leading terms of the large- $N_{f}$ expansion. This procedure transforms a large- $N_{f}$ expansion into a large- $\beta_{0}$ expansion, which in addition to quark bubbles insertions into the renormalon chain, is taking into account the contributions of the gluon- and ghost-bubbles insertions as well (though neglecting definite one-loop insertions into the gluon-quark-antiquark vertex, which should be also considered in the process of rigorous calculation of the coefficient $\beta_{0}$ ). The application of the NNA approach allowed the authors of Ref. [28] to formulate the extension to higher orders of the BLM-approach [29]. Technically, the work of Ref. 28. supports the results of the first successful formulation of the BLM procedure to the next-to-next-to-leading order [30] Moreover, these two works pushed ahead the study of the BLM procedure in higher orders [31. In principle, the relations of the results of Refs. [30, 28, 31] need more detailed considerations. In view of the lack of space we will avoid discussions of this subjects here.

The Borel images calculated by this procedure for the GLS and Bjp sum rules coincide and have the following form $[9]$ :

$$
B\left[\mathrm{C}_{\mathrm{Bjp}}\right](\delta)=\mathrm{B}\left[\mathrm{C}_{\mathrm{GLS}}\right](\delta)=-\frac{(3+\delta) \exp (5 \delta / 3)}{\left(1-\delta^{2}\right)\left(1-\delta^{2} / 4\right)} .
$$


They contain the IRR poles at $\delta=1$ and $\delta=2$ and the UVR poles at $\delta=-1$ and $\delta=-2$. Note that the $\delta=-1$ UVR poles in Eq. (27) are suppressed by a factor $(1 / 2) \exp (-10 / 3)=$ 0.018 , relative to the dominant IRR poles at $\delta=1$ [10]. Therefore, in the asymptotic structure of the perturbative QCD effects in the expressions for $C_{\mathrm{GLS}}\left(Q^{2}\right) \approx C_{B j p}\left(Q^{2}\right)$ (where we neglect the small "light-by-light-type" effects, contributing to $C_{\mathrm{GLS}}\left(Q^{2}\right)$ ) the sign-constant part in Eq. (5) dominates strongly with respect to the sign-alternating contribution, generated by $\delta=-1$ UVR. The scheme-dependence of these results are not so obvious, Indeed, the suppressions of $\delta=1 \mathrm{UVR}$ with respect to $\delta=1 \mathrm{IRR}$ is related to the application of the $\overline{\mathrm{MS}}$-scheme which we are using throughout the whole work. In fact in this scheme the IRR renormalons are not suppressed. However, there is the procedure, when the situation is reversed- the IRR are absent, but UVR may exist. This feature is manifesting itself for the models with "frozen" coupling constant (see e.g. [33] ).

Returning to the large- $N_{f}$ expansion of the perturbative expressions

$$
C_{\mathrm{Bjp}}\left(Q^{2}\right)=C_{G L S}\left(Q^{2}\right)=1+\frac{C_{F}}{T_{f} N_{f}} \sum_{n=1}^{\infty} r_{n}\left(T_{f} N_{f} a_{s}\right)^{n}
$$

where $C_{F}=4 / 3, T_{f}=1 / 2$ and

$$
r_{n}=\lim _{\delta \rightarrow 0}\left(-\frac{4}{3} \frac{d}{d \delta}\right)^{n-1} B\left[C_{\mathrm{Bjp}}\right](\delta),
$$

we arrive at the following expansion in powers of $x=T_{f} N_{f} a_{s}$, namely

$$
\sum_{n} r_{n} x^{n}=-3 x+8 x^{2}-\frac{920}{27} x^{3}+\frac{38720}{243} x^{4}+\ldots \quad,
$$

which is known in the $\overline{\mathrm{MS}}$ scheme up to $O\left(\alpha_{s}^{9} N_{f}^{9}\right)$ terms 9 . Using now the traditional $\overline{\mathrm{MS}}$-scheme expansion in terms of the orders in $\alpha_{s} / \pi=4 a_{s}$, one can compare the results of explicit perturbative calculations of

$$
C_{\mathrm{Bjp}}\left(Q^{2}\right)=1+\sum_{n \geq 1} r_{n}\left(\frac{\alpha_{s}}{\pi}\right)^{n}
$$

with the known numbers

$$
\begin{aligned}
& r_{1}=-1 \\
& r_{2}=-4.5833+0.33333 N_{f} \\
& r_{3}=-41.440+7.6073 N_{f}-0.17747 N_{f}^{2}
\end{aligned}
$$

obtained at $O\left(\alpha_{s}^{2}\right)$ in Ref. [13] and at $O\left(\alpha_{s}^{3}\right)$ in Ref. [14, with the results of the application of the NNA procedure 27] to the estimates of the perturbative QCD corrections from large$N_{f}$ expansion of Eq. (30) ${ }^{3}$. Performing the shift $N_{f} \rightarrow N_{f}-33 / 2$ in the second, third and fourth terms in Eq. (30), we arrive at the following estimates in the $\overline{\mathrm{MS}}$ scheme [10]:

$$
\begin{aligned}
& r_{2}^{\mathrm{NNA}}=-5.5+0.33333 N_{f} \\
& r_{3}^{\mathrm{NNA}}=-48.316+5.8565 N_{f}-0.17747 N_{f}^{2} \\
& r_{4}^{\mathrm{NNA}}=-466.00+84.728 N_{f}-5.1350 N_{f}^{2}+0.10374 N_{f}^{3} .
\end{aligned}
$$

\footnotetext{
${ }^{3}$ It is worth noting that similar NNA analysis was performed previously, in Ref. [32], for the $e^{+} e^{-}$ annihilation Adler $D$-function.
} 
Reasonable agreement can be observed between the sign structure and values of the NNA estimates and the results of explicit calculations (compare the estimates of Eqs. (35) and (36) with the numbers in Eqs. (33) and (34), respectively). As to the prediction for $r_{4}^{\mathrm{NNA}}$, it may serve as a guide for understanding the rate of growth of the coefficients of the perturbative series generated by the single renormalon-chain approximation.

Consider now the Bjunp sum rule, which is defined in Eq. (23). Within the large- $N_{f}$, expansion its perturbative coefficient function

$$
C_{\text {Bjpunp }}\left(Q^{2}\right)=1+\sum_{n \geq 1} \tilde{r}_{n}\left(\frac{\alpha_{s}}{\pi}\right)^{n}
$$

was calculated in the $\overline{\mathrm{MS}}$ scheme and large- $N_{f}$ expansion up to a $O\left(\alpha_{s}^{9} N_{f}^{9}\right)$-terms [10]. Following the logic of our work, we present here the results for the first 4 terms only:

$$
\sum_{n} \tilde{r}_{n} x^{n}=-2 x+\frac{64}{9} 8 x^{2}-\frac{2480}{81} x^{3}+\frac{113920}{729} x^{4}+\ldots
$$

As was already mentioned above, the explicit results of calculations of the perturbative contributions to the Bjunp sum rule

$$
C_{\text {Bjunp }}\left(Q^{2}\right)=1+\sum_{n \geq 1} \tilde{r}_{n}\left(\frac{\alpha_{s}}{\pi}\right)^{n}
$$

are known up to the order $O\left(\alpha_{s}^{3}\right)$ level. These results are:

$$
\begin{aligned}
& \tilde{r}_{1}=-2 / 3 \\
& \tilde{r}_{2}=-3.8333+0.29630 N_{f} \\
& \tilde{r}_{3}=-36.155+6.3313 N_{f}-0.15947 N_{f}^{2}
\end{aligned}
$$

where $\tilde{r}_{2}$ was calculated in Ref. [25] while $\tilde{r}_{3}$ was evaluated in Ref. [26]. Applying now the NNA procedure to the series of Eq. (39), we find that, in the $\overline{\mathrm{MS}}$ scheme, the estimated coefficients of the Bjunp sum rules have the following form [10]:

$$
\begin{aligned}
& \tilde{r}_{2}^{\mathrm{NNA}}=-4.8889+0.29630 N_{f} \\
& \tilde{r}_{3}^{\mathrm{NNA}}=-43.414+5.2623 N_{f}-0.15947 N_{f}^{2} \\
& \tilde{r}_{4}^{\mathrm{NNA}}=-457.02+83.094 N_{f}-5.0360 N_{f}^{2}+0.10174 N_{f}^{3} .
\end{aligned}
$$

The estimate of Eq. (44) is in agreement with its exact partner of Eq. (42). The same situation holds for the $O\left(\alpha_{s}^{3}\right)$ corrections (compare Eq. (45) with Eq. (43)). It should be stressed, that the similarity of the next-to-next-to- leading-order $\overline{\mathrm{MS}}$-scheme perturbative QCD contributions to the Bjp and Bjunp sum rules was previously noticed in Ref. 34], although no explanation of this observation was given. Now, within the NNA procedure, it is possible to generalize this observation to higher-order level. Indeed, the NNA estimates of the $O\left(\alpha_{s}^{4}\right)$ corrections to the Bjp and Bjunp sum rules have a similar expressions as well. These facts may indicate the close similarity in the full perturbative structure of the QCD corrections to the Bjunp sum rule, the Bjp sum rule and the GLS 
sum rule (provided the "light-by-light-type" terms will not drastically modify the values of perturbative terms in the latter case in the one-renormalon chain approximation). Note that, generally speaking, from this order of perturbation theory the diagrams from the second renormalon chain are starting to contribute to the quantities under consideration. These diagrams may influence the asymptotic behavior of the the series considered [35. In view of this it seems that it is more rigorous to use, in the phenomenological application, the order of $\alpha_{s}^{4}$-terms, estimated in Ref. 36] using the PMS approach [37] and the effectivecharges approach, developed in Ref. 38. However, since in this work we concentrated ourselves on the structure of the QCD expressions, obtained in the one-renormalon chain approximation, we will avoid more detailed discussions of the possible influence of the multi-renormalon chain contributions to the results of our studies.

The observed in Ref. 34] similarity of the next-to-next-to-leading-order approximations for the Bjp and Bjunp sum rules was attributed in Ref. [10] to the fact that the dominant $\delta=1$ IRR contribution to the Borel images of these sum rules enters with identical residues. Indeed, the Borel images in the Borel integrals of Eq. (7) for the Bjunp and Bjp sum rules turn out to be closely related 10 , namely

$$
\left.B\left[C_{\mathrm{Bjunp}}\right](\delta)=\left(\frac{2(1+\delta)}{3+\delta}\right) B\left[C_{\mathrm{Bjp}}\right](\delta)=-\frac{2 \exp (5 \delta / 3)}{(1-\delta)\left(1-\delta^{2} / 4\right)}\right)
$$

Comparing Eq. (27) with Eq. (47) one can convince oneself that the residues of the poles at $\delta=1$ in these two expressions are really the same and are equal to the factor $-(8 / 3) \exp (5 / 3)$.

Notice also the absence of $\delta=-1$ UVR pole and the existence in Eq. (47) of a $\delta=-2$ UVR pole together with the leading $\delta=1$ IRR one. Thus we are observing one more interesting fact: the structure of the Borel image for the Borel sum, related to the Bjunp sum rule, is dual to the structure of leading renormalon contributions to the Borel image of the Borel sum for the $e^{+} e^{-}$annihilation Adler D-function. Indeed, in the latter case the leading IRR is manifesting itself at $\delta=2$, while the leading UVR pole is appearing at $\delta=-1$ (the general structure of renormalon singularities in the $e^{+} e^{-}$ annihilation channel was analyzed in Ref. [1], while the concrete $\overline{\mathrm{MS}}$-scheme calculations of the corresponding Borel image were done later on in Refs. [39] and 40]).

The absence of $\delta=1$ IRR in the Borel sum of the $e^{+} e^{-}$annihilation channel is related to the absence of $O\left(\Lambda^{2} / Q^{2}\right)$ non-perturbative power correction in the standard variant of the operator product expansion formalism, applied to the theoretical expression for the $e^{+} e^{-}$annihilation Adler D-function. Indeed, the existence of lowest dimension-4 quark and gluon condensates [41] in this channel can be associated in terms of renormalon language with the existence of the leading IRR pole, which in case of "Borelization" of the Adler Dfunction is appearing at $\delta=2$. However, as was already discussed above, the dimension-2 non-perturbative corrections enter into the theoretical expressions for the three DIS sum rules we are interested in. In the IRR language, this corresponds to the appearance of a $\delta=1$ IRR pole 3], which manifests itself in the concrete results of Refs. [9], [10] (see Eqs. (27) and (47)). Thus, it should be stressed that the structure of singularities of the Borel sums (or images) is not universal and depends from the physical quantity under consideration. 


\section{IRR for DIS sum rules and the values of twist-4 corrections}

In addition to controlling the sign-positive $n$ ! growth of the asymptotic series the existence of $\delta=1$ IRR gives an ambiguity in taking the Borel integral of Eq. (7) over this pole. In the case of large $\beta_{0}$ expansion and for the series we are interested in, this ambiguity was estimated in Ref. [5]. Moreover, $\delta=1$ IRR generates the negative power suppressed correction which has the following expression:

$$
\Delta C_{\text {sum rules }} \approx-\frac{32 \exp (5 / 3)}{9 \beta_{0}} \frac{\Lambda \frac{2}{\mathrm{MS}}}{Q^{2}} .
$$

Notice, that it has the same negative sign as the residue of $\delta=1 \mathrm{IRR}$.

This estimate may be coordinated with the definition of the twist-4 matrix element in the sum rules we are interested in. Therefore, we will make the assumption that the identical values and signs of the IRR induced power-suppressed term indicate that the values of twist- 4 contributions to the expressions of GLS, Bip and Bjunp sum rules, normalized to unity, should have the same negative sign and a similar closed value [11. This assumption is similar to the known in the renormalon-oriented literature guess of "universality" 42].

Let us check this assumption, considering the following expressions for the sum rules we are interested in

$$
\begin{aligned}
\operatorname{GLS}\left(Q^{2}\right) & =3\left[1-4 a_{s}-O\left(a_{s}^{2}\right)-\frac{\mathrm{A}}{Q^{2}}\right], \\
\operatorname{Bjp}\left(Q^{2}\right) & =\frac{g_{A}}{6}\left[1-4 a_{s}-O\left(a_{s}^{2}\right)-\frac{\mathrm{B}}{Q^{2}}\right], \\
\operatorname{Bjunp}\left(Q^{2}\right) & =\left[1-\frac{8}{3} a_{s}-O\left(a_{s}^{2}\right)-\frac{\mathrm{C}}{Q^{2}}\right],
\end{aligned}
$$

where $\mathrm{A}=\left\langle\left\langle O_{1}\right\rangle\right\rangle / 3, \mathrm{~B}=\left\langle\left\langle O_{2}\right\rangle\right\rangle\left(6 / g_{A}\right)$ and $\mathrm{C}=\left\langle\left\langle O_{3}\right\rangle\right\rangle$ and compare in Table 1 the results of different theoretical and phenomenologically based evaluations of the twist-4 parameters $\mathrm{A}, \mathrm{B}$ and $\mathrm{C}$.

In the case of the GLS and Bjunp sum rules the results of the original application of the three-point function QCD sum rules method gave $\left\langle\left\langle O^{\mathrm{S}}\right\rangle\right\rangle=0.33 \mathrm{GeV}^{2}$ and $\left\langle\left\langle O^{\mathrm{NS}}\right\rangle\right\rangle=$ $0.15 \mathrm{GeV}^{2}$, with over $50 \%$ error bars 43 , while the three-point function estimates for the modified results of calculations of the twist-4 parameter of the Bjp sum rule resulted in the following value $M_{N}^{2} f_{2}^{p-n}=-0.18 \pm 0.09 \mathrm{GeV}^{2}$ in the region where nucleon target mass corrections of $O\left(M_{N}^{2} / Q^{2}\right)$ and twist-3 contribution may be neglected [20]. As was already mentioned, these calculations were re-examined using three-point function QCD sum-rules approach in Refs. 21] and [22. In the first case the obtained result turned out to be larger than the original results from Ref. [20] and has the following value of $M_{N}^{2} f_{2}^{p-n}=-0.634 \pm 0.317 \mathrm{GeV}^{2}$ [21], while in the latter case it was considerably smaller,

namely $M_{N}^{2} f_{2}^{p-n}=-0.07 \pm 0.035$ [22], although within $50 \%$ theoretical uncertainty we adopt for all calculations within three-point function QCD sum-rules approach, this value 
Table 1: The results for twist-4 contributions to the GLS, Bjp and Bjunp sum-rule expressions of Eqs. (49)-(51).

\begin{tabular}{|l|c|c|c|}
\hline & $\mathrm{A}\left[\mathrm{GeV}^{2}\right]$ & $\mathrm{B}\left[\mathrm{GeV}^{2}\right]$ & $\mathrm{C}\left[\mathrm{GeV}^{2}\right]$ \\
\hline \hline QCD sum rules (Ref.[43]) & $0.098 \pm 0.049$ & - & $0.133 \pm 0.065$ \\
QCD sum rules (Ref.[20] & - & $0.063 \pm 0.031$ & - \\
QCD sum rules (Ref.[21] & $0.158 \pm 0.078$ & $0.223 \pm 0.118$ & $0.16 \pm 0.08$ \\
QCD sum rules (Ref.[22]) & - & $0.025 \pm 0.012$ & - \\
Instanton model (Ref.[46]) & $0.078 \pm 0.039$ & $0.087 \pm 0.043$ & - \\
Instanton model (Ref.[47]) & - & - & $0.16 \pm 0.08$ \\
Experiment (Ref.[48]) & -- & $0.098 \pm 0.028$ & - \\
Experiment (Ref.[50]) & $0.04 \pm 0.13$ & - & - \\
\hline
\end{tabular}

does not disagree with the results obtained in Ref. 20]. The relatively high difference between the central values of estimates of Refs. 21] and [20] is explained by the fact that in the former analysis the additional corrections to the perturbative side of the corresponding QCD sum rules are included and the continuum term to the nucleon pole of the low-energy side of this sum rule is explicitly retained. This leads to better stability of the extracted value of matrix element with respect to the Borel parameter and increases its central value. Note, however, that the theoretical error of the three-point function of the QCD sum rules result of Ref. [21] is considerably underestimated. We fix it as $50 \%$ uncertainty, which to our point of view is typical to all three-point function QCD sum rules results.

In Table 1 we present the estimates of twist- 4 corrections to different DIS sum rules, obtained with the help of the three-point function QCD sum-rules approach and compare them with the results of the application of different theoretical approach, based on the picture of the QCD vacuum as a "medium" of instantons 44. This picture was further developed in the method in Ref. 45] and applied for estimating twist-4 contributions to the GLS sum rule and Bjp sum rule in Ref. 46], while the number for the twist-4 contribution to the Bjunp sum rule, which follows from this approach, was presented in Ref. 47]. In the absence of estimates of theoretical uncertainties within this approach, we will apply to them the careful $50 \%$ estimate as well. All these results support the original results of the three-point function QCD sum rules calculations of the twist- 4 corrections to the GLS, Bjunp sum rules 43] and Bjp sum rule[20, though the additional three-point function QCD sum rules cross-check of the results of Ref. [20] may be rather useful.

The experimentally motivated value of the twist-4 contribution to the Bjorken sum rule $M_{n}^{2} f_{2}^{p-n}=-0.28 \pm 0.08 \mathrm{GeV}^{2}$ [48] was obtained by means of integrating in $x$ the numerator of the dimensionless $h(x) / Q^{2}$ contributions, extracted from the fits of world average data for $g_{1}^{p}\left(x, Q^{2}\right)$ and $g_{1}^{n}\left(x, Q^{2}\right)$ performed in Ref. [49]. From the results of Table 1 one can see that the agreement with the QCD sum-rules calculations of Ref. [20] and instanton-based calculations of Ref. 46] is more than qualitative.

The experimentally inspired estimate for the twist- 4 contribution to the GLS sum rule was obtained only recently [50] as a result of the integration of $x$-dependence of the twist-4 contribution $h(x) / Q^{2}$, extracted in the works of Ref. [51] devoted to the analysis of $x F_{3}$ data 
of CCFR collaboration. One can see that the central value of the contribution is negative (in fact it comes with the negative sign in the sum rule) but has rather large uncertainties. So, at the present level we cannot obtain from this estimate even qualitative information and additional work on its improvement is needed.

To conclude, we present the final results for the GLS, Bjp and Bjunp sum rules, where for definiteness the twist-4 matrix elements are estimated using the central values of the three-point function QCD sum-rules results from Refs. 43] and [20]:

$$
\begin{aligned}
\operatorname{GLS}\left(Q^{2}\right) & =3\left[1-4 a_{s}-O\left(a_{s}^{2}\right)-\frac{0.098 \mathrm{GeV}^{2}}{Q^{2}}\right], \\
\operatorname{Bjp}\left(Q^{2}\right) & =\frac{g_{A}}{6}\left[1-4 a_{s}-O\left(a_{s}^{2}\right)-\frac{0.063 \mathrm{GeV}^{2}}{Q^{2}}\right], \\
\operatorname{Bjunp}\left(Q^{2}\right) & =\left[1-\frac{8}{3} a_{s}-O\left(a_{s}^{2}\right)-\frac{0.133 \mathrm{GeV}^{2}}{Q^{2}}\right] .
\end{aligned}
$$

It should be stressed that they all have the same negative sign and within existing theoretical uncertainties are in agreement with each other. This fact was anticipated by the identical value of the ambiguity, generated by the $\delta=1$ IRR pole of the Borel images of all these three sum rules (see Eq. (48)). Moreover, as follows from the results of application of the single-renormalon chain approximation in the perturbative sector presented in Sec.2, we may expect a similar asymptotic behavior of the perturbative corrections to all these three sum rules (compare Eqs.(33)-(36) with Eqs.(42)-(45)). It is interesting that the similar property is manifesting itself in perturbative series under investigations at the $O\left(\alpha_{s}^{3}\right)$ level, studied within scheme-invariant approaches in Ref. 36.

These facts give us the idea that the sum rules we are interested in are closely related and that, in the region, where we can neglect target mass corrections and twist-3 contributions to the Bjp sum rule and quark-mass dependent corrections (say in the region $Q^{2} \geq 2 \mathrm{GeV}^{2}$ ) we can write down the following basic relation [1]:

$$
\operatorname{Bjp}\left(Q^{2}\right) \approx\left(g_{A} / 18\right) \operatorname{GLS}\left(Q^{2}\right) \approx\left(g_{A} / 6\right) \operatorname{Bjunp}\left(Q^{2}\right)
$$

In the next section we will present more detailed considerations of the experimental consequences of these relations then those, that are briefly outlined in Ref. 11].

\section{IRR- inspired relations and experiment}

In order to test whether our basic relation Eq. (55) is respected by experiment, we first present the results of the extraction of the GLS sum rule by combining CCFR neutrino DIS data with the data for other neutrino DIS experiments for $1 \mathrm{GeV}^{2}<Q^{2}<15 \mathrm{GeV}^{2}$ 52. It is known that the weighted extraction of $\alpha_{s}\left(M_{Z}\right)$ from these data result in the rather rough value $\alpha_{s}\left(M_{Z}\right)=0.115 \pm_{0.12}^{0.009}$, which, is in agreement with $\alpha_{s}\left(M_{Z}\right)=0.115 \pm$

0.001 (stat) \pm 0.005 (syst) \pm 0.003 (twist) \pm 0.0005 (scheme), extracted in Ref. 53 . from the previous CCFR data for the GLS sum rule at $Q^{2}=3 \mathrm{GeV}^{2}[54$. However, for our 
Table 2: The results for the GLS sum rule from Ref. [52]

\begin{tabular}{|l|c|}
\hline$Q^{2}\left[\mathrm{GeV}^{2}\right]$ & GLS sum rule \\
\hline \hline 2.00 & $2.49 \pm 0.08 \pm 0.14$ \\
3.16 & $2.55 \pm 0.08 \pm 0.10$ \\
5.01 & $2.78 \pm 0.06 \pm 0.19$ \\
7.94 & $2.82 \pm 0.07 \pm 0.19$ \\
12.59 & $2.80 \pm 0.13 \pm 0.18$ \\
\hline
\end{tabular}

Table 3: The comparison of the results of application of Eq. (55) with direct experimentally motivated numbers

\begin{tabular}{|l|c|c|}
\hline$Q^{2}\left[\mathrm{GeV}^{2}\right]$ & Bjp from Table 1 & Bjp SR (exp) \\
\hline \hline 2.00 & $0.174 \pm 0.006 \pm 0.010$ & $0.169 \pm 0.025$ [Ref.[55]] \\
3.16 & $0.178 \pm 0.004 \pm 0.007$ & $0.164 \pm 0.023$ [Ref. [55]] \\
5.01 & $0.195 \pm 0.004 \pm 0.013$ & $0.181 \pm 0012$ (stat) \pm 0.018 (syst) [Ref.[56]] \\
7.94 & $0.197 \pm 0.005 \pm 0.013$ & $\overline{\text { [56. }}$ \\
12.5 & $0.196 \pm 0.009 \pm 0.013$ & $0.195 \pm 0.029$ Ref. [57] \\
\hline
\end{tabular}

purposes we will not need to re-extract $\alpha_{s}\left(M_{Z}\right)$ values from the GLS sum rule results of Ref. [52], but will use these, which are presented in Table 2.

To estimate the values of the Bjp sum rule from the results of Table 2 we will use our main equation (55) and will compare them with available experimental data for the Bjp sum rule. The results of these studies are presented in Table 3.

One can see that though the central values of estimated numbers for the Bjp SR are higher than the results of the SLAC E143 collaboration [55], they agree within error bars. It is also interesting to compare the result from Table 3 with the value of the Bjp sum rule extracted in Ref. 58 from the SLAC and SMC data $\operatorname{Bjp}\left(3 \mathrm{GeV}^{2}\right)=0.177 \pm 0.018$ and which, within error bars, do not contradict the value $\operatorname{Bjp}\left(3 \mathrm{GeV}^{2}\right)=0.164 \pm 0.011$ used in the work of Ref. [59]. It is rather inspiring that within error bars these results agree with the GLS sum rule value at $Q^{2}=3.16 \mathrm{GeV}^{2}$. The same feature holds for the Bjp sum rules at $Q^{2}=5 \mathrm{GeV}^{2}$, namely for the SMC result of Ref. [56]. Thus we think that within existing uncertainties our approximate IRR-inspired basic equation (55) is supported by existing experimental data.

\section{Conclusions}

We demonstrated that the existing phenomenological data do not contradict the basic relation of Eq. (55) and therefore the reliability of the one-renormalon chain approximation of the theoretical quantities under consideration. For its more detailed studies, we may rely on the appearance of Neutrino Factory data for all sum rules, which enter in Eq. (55). In fact it may provide rather useful data not only for the GLS and 
Bjp sum rules, but for the Bjunp sum rule as well (for a discussion of this possibility see Refs. 60, 61] ).

Another interesting option of the relation of Eq. (55) is to analyze the sources of its possible violation in the lower energy region of over $Q^{2} \approx 1 \mathrm{GeV}^{2}$, where one may compare the CCFR data for the GLS sum rule at the energy point $Q^{2}=1.26 \mathrm{GeV}^{2} 52$ and the JLAB data for the Bjp sum rule at $Q^{2}=1.10 \mathrm{GeV}^{2}[23]$.

To conclude this section, we would like to emphasize that the problems considered by us in this work are complementary to the considerations of Ref. 62. In the former analysis, the GLS and Bjp sum rules were determined in the high energy point of over $Q^{2}=12.33 \mathrm{GeV}^{2}$ from the generalized Crewther relation constructed in 9, 62, 63, using the extension of the BLM approach of Ref. 29] and the analysis of $e^{+} e^{-}$annihilation data from Ref. 64. Certainly, the renormalon-chain insertions are absorbed in this approach into the BLM scale. However, the considerations within this language of the high-twist effects is still missed. It may be of interest to think of the possibility of evaluating hightwist contributions to the Crewther relation, which relates, in the Eucledian region we are working massless QCD perturbative contributions to the Adler D-function of $e^{+} e^{-}$annihilation with the perturbative corrections to the GLS and Bjp sum rules.

Acknowledgements I am grateful to D.J. Broadhurst for a productive collaboration. It is a pleasure to express my personal thanks to S.I. Alekhin, G. Altarelli, Yu.L. Dokshitzer, J. Ellis, G. Grunberg, A.V. Sidorov and V.I. Zakharov for useful discussions at various stages of this work. This article grew up from my talk at the 19th Rencontre de Physique de la Vallèe d'Aoste (27 February-5 March,2005, La Thuile, Aosta Valley, Italy). I would like to thank its organizers, G. Bellettini and M. Greco for their invitation. This work is supported by RFBR Grants N03-02-17047, 03-02-17177 and N 05-01-00992. It was continued during the visit to CERN. I have real pleasure in thanking the members of the CERN Theory Group for hospitality. In its final form the work was completed during the visit to ICTP (Trieste). I am grateful to a referee for constructive advise

\section{References}

[1] V. I. Zakharov, Nucl. Phys. B 385, 452 (1992).

[2] A. H. Mueller, CU-TP-573, Proc. Int. Conference "QCD-20 Years Later" Ed. by P.M. Zerwas and H.A. Kastrup (World Scientific, 1992) pp.162-171.

[3] A. H. Mueller, Phys. Lett. B 308, 355 (1993).

[4] M. Beneke, Phys. Rep. 317, 1 (1999) arXiv:hep-ph/9807443.

[5] M. Beneke and V. M. Braun, In the "Boris Ioffe Festschrift : At the Frontiers of Particle Physics/Handbook of QCD", Ed. by M. Shifman (World Scientific, 2001), pp. 1719-1773 arXiv:hep-ph/0010208.

[6] G. Altarelli, CERN/TH-95-309, Proc. Int. School of Subnuclear Physics: 33rd Course "Vacuum and Vacua: The Physics of Nothing", Ed. A. Zichichi, 1995, pp. 221-248. 
[7] R. B. Dingle, Asymptotic expansions: their derivation and interpretation, Ch. XXI (Academic Press, London and New York, 1973).

[8] M. Beneke, V. M. Braun and N. Kivel, Phys. Lett. B 443, 308 (1998) arXiv:hep-ph/9809287.

[9] D. J. Broadhurst and A. L. Kataev, Phys. Lett. B 315, 179 (1993) arXiv:hep-ph/9308274.

[10] D. J. Broadhurst and A. L. Kataev, Phys. Lett. B 544, 154 (2002) arXiv:hep-ph/0207261.

[11] A. L. Kataev, Pisma Zhetf. 81, 744 (2005), arXiv:hep-ph/0505108.

[12] D. J. Gross and C. H. Llewellyn Smith, Nucl. Phys. B 14, 337 (1969).

[13] S. G. Gorishny and S. A. Larin, Phys. Lett. B 172, 109 (1986).

[14] S. A. Larin and J. A. M. Vermaseren, Phys. Lett. B 259, 345 (1991).

[15] E. V. Shuryak and A. I. Vainshtein, Nucl. Phys. B 199, 451 (1982).

[16] J. D. Bjorken, Phys. Rev. 148, 1467 (1966).

[17] E. V. Shuryak and A. I. Vainshtein, Nucl. Phys. B 201, 141 (1982).

[18] X. D. Ji and P. Unrau, Phys. Lett. B 333, 228 (1994) arXiv:hep-ph/9308263.

[19] I. Hinchliffe and A. Kwiatkowski, Annu. Rev. Nucl. Part. Sci. 46, 609 (1996) arXiv:hep-ph/9604210.

[20] I. I. Balitsky, V. M. Braun and A. V. Kolesnichenko, Phys. Lett. B 242245 (1990) [Erratum-ibid. B 318, 648 (1993)] arXiv:hep-ph/9310316.

[21] G. G. Ross and R. G. Roberts, Phys. Lett. B 322, 425 (1994) arXiv:hep-ph/9312237.

[22] E. Stein, P. Gornicki, L. Mankiewicz, A. Schafer and W. Greiner, Phys. Lett. B 343, 369 (1995) arXiv:hep-ph/9409212.

[23] A. Deur et al., Phys. Rev. Lett. 93, 212001 (2004) arXiv:hep-ex/0407007.

[24] J. D. Bjorken, Phys. Rev. 163, 1767 (1967).

[25] K. G. Chetyrkin, S. G. Gorishny, S. A. Larin and F. V. Tkachov, Phys. Lett. B 137, 230 (1984).

[26] S. A. Larin, F. V. Tkachov and J. A. M. Vermaseren, Phys. Rev. Lett. 66, 862 (1991).

[27] D. J. Broadhurst and A. G. Grozin, Phys. Rev. D 52, 4082 (1995) arXiv:hep-ph/9410240.

[28] M. Beneke and V. M. Braun, Phys. Lett. B 348513 (1995) arXiv:hep-ph/9411229. 
[29] S. J. Brodsky, G. P. Lepage and P. B. Mackenzie, Phys. Rev. D 28, 228 (1983).

[30] G. Grunberg and A. L. Kataev, Phys. Lett. B 279 (1992) 352.

[31] S. V. Mikhailov, arXiv:hep-ph/0411397.

[32] C. N. Lovett-Turner and C. J. Maxwell, Nucl. Phys. B 452, 188 (1995) arXiv:hep-ph/9505224.

[33] D. V. Shirkov and I. L. Solovtsov, Phys. Rev. Lett. 79, 1209 (1997) arXiv:hep-ph/9704333.

[34] E. Gardi and M. Karliner, Nucl. Phys. B 529, 383 (1998) arXiv:hep-ph/9802218.

[35] A. I. Vainshtein and V. I. Zakharov, Phys. Rev. Lett. 73, 1207 (1994) [Erratum-ibid. 75, 3588 (1995)] arXiv:hep-ph/9404248.

[36] A. L. Kataev and V. V. Starshenko, Mod. Phys. Lett. A 10, 235 (1995) arXiv:hep-ph/9502348.

[37] P. M. Stevenson, Phys. Rev. D 23, 2916 (1981).

[38] G. Grunberg, Phys. Rev. D 29, 2315 (1984).

[39] M. Beneke, Nucl. Phys. B 405, 424 (1993).

[40] D. J. Broadhurst, Z. Phys. C 58, 339 (1993).

[41] M. A. Shifman, A. I. Vainshtein and V. I. Zakharov, Nucl. Phys. B 147, 385 (1979).

[42] Y. L. Dokshitzer, G. Marchesini and B. R. Webber, Nucl. Phys. B 469, 93 (1996) arXiv:hep-ph/9512336.

[43] V. M. Braun and A. V. Kolesnichenko, Nucl. Phys. B 283, 723 (1987).

[44] E. V. Shuryak, Nucl. Phys. B 203, 93 (1982).

[45] D. Diakonov and V. Y. Petrov, Nucl. Phys. B 245, 259 (1984).

[46] J. Balla, M. V. Polyakov and C. Weiss, Nucl. Phys. B 510, 327 (1998) arXiv:hep-ph/9707515.

[47] C. Weiss, J. Phys. G 29, 1981 (2003) [ arXiv:hep-ph/0210132.

[48] A. V. Sidorov and C. Weiss, arXiv:hep-ph/0410253.

[49] E. Leader, A. V. Sidorov and D. B. Stamenov, Phys. Rev. D 67, 074017 (2003) arXiv:hep-ph/0212085.

[50] A. V. Sidorov, private communication. 
[51] A. L. Kataev, A. V. Kotikov, G. Parente and A. V. Sidorov, Phys. Lett. B 417, 374 (1998) arXiv:hep-ph/9706534;

A. L. Kataev, G. Parente and A. V. Sidorov, Nucl. Phys. B 573, 405 (2000) arXiv:hep-ph/9905310;

A. L. Kataev, G. Parente and A. V. Sidorov, Phys. Part. Nucl. 34, 20 (2003) [Fiz. Elem. Chast. Atom. Yadra 34, 43 (2003)] arXiv:hep-ph/0106221.

[52] J. H. Kim et al., Phys. Rev. Lett. 81, 3595 (1998) arXiv:hep-ex/9808015.

[53] J. Chyla and A. L. Kataev, Phys. Lett. B 297, 385 (1992) arXiv:hep-ph/9209213.

[54] W. C. Leung et al., Phys. Lett. B 317, 655 (1993).

[55] K. Abe et al. [E143 collaboration], Phys. Rev. D 58, 112003 (1998) arXiv:hep-ph/9802357.

[56] B. Adeva et al. [Spin Muon Collaboration], Phys. Rev. D 58, 112002 (1998).

[57] B. Adeva et al. [Spin Muon Collaboration (SMC)], Phys. Lett. B 412, 414 (1997).

[58] G. Altarelli, R. D. Ball, S. Forte and G. Ridolfi, Nucl. Phys. B 496, 337 (1997) arXiv:hep-ph/9701289.

[59] J. R. Ellis, E. Gardi, M. Karliner and M. A. Samuel, Phys. Lett. B 366, 268 (1996) arXiv:hep-ph/9509312.

[60] M. L. Mangano et al., arXiv:hep-ph/0105155.

[61] S. I. Alekhin and A. L. Kataev, J. Phys. G 29, 1993 (2003) arXiv:hep-ph/0209165.

[62] S. J. Brodsky, G. T. Gabadadze, A. L. Kataev and H. J. Lu, Phys. Lett. B 372, 133 (1996) arXiv:hep-ph/9512367.

[63] R. J. Crewther, Phys. Rev. Lett. 28, 1421 (1972) and Phys. Lett. B 397, 137 (1997) arXiv:hep-ph/9701321.

[64] A. C. Mattingly and P. M. Stevenson, Phys. Rev. D 49, 437 (1994) arXiv:hep-ph/9307266. 